\title{
Propiedades psicométricas de la Escala de Impulsividad (UPPS-P) en una muestra de adultos argentinos
}

\author{
Psychometric properties of the Impulsivity Scale (UPPS-P)
}

in a sample of Argentine adults

\author{
Karen Pinter ${ }^{1}$, Pablo Christian González Caino ${ }^{2}$ y Santiago Resett ${ }^{3}$ \\ ${ }^{1}$ Licenciada en Psicología. Universidad Argentina de la Empresa. \\ E-mail: karenpinter@gmail.com
}

${ }^{2}$ Doctor en Psicología. Becario posdoctoral del Consejo Nacional de Investigaciones Científicas

y Técnicas (CONICET) en la Universidad Argentina de la Empresa (UADE). Investigador y docente de la Universidad Argentina de la Empresa. E-mail: pablo.cg.caino@hotmail.com ${ }^{3}$ Doctor en Psicología. Investigador Asistente del CONICET. Investigador y docente de la

Universidad Argentina de la Empresa. E-mail: santiago_resett@hotmail.com

\author{
Universidad Argentina de la Empresa. \\ Ciudad Autónoma de Buenos Aires (CABA), Argentina.
}

\section{Resumen}

Este estudio tiene como objetivo explorar las propiedades psicométricas de la Escala de Impulsividad (UPPS-P) en su versión breve en una muestra de adultos argentinos. Se exploró su estructura factorial y consistencia interna, y su validez convergente con los estilos de pensamiento experiencial y racional, así como con la psicopatía. A su vez, se exploró la relación de la impulsividad con el narcisismo y maquiavelismo, controlando los puntajes en psicopatía. Para ello, se seleccionó una muestra intencional de adultos argentinos $(\mathrm{n}=517)$ de entre 18 y 60 años ( $54 \%$ femenino, edad media 27), reclutados mediante diversas redes sociales. Los participantes respondieron de forma online a dicha escala, a un inventario sociodemográfico, así como al Inventario Experiencial Racional y a la Escala de Personalidad Oscura. Los resultados obtenidos se procesaron con el programa SPSS 23, Factor 8.10 y AMOS 16.0. Un análisis en paralelo, sumado a análisis factoriales confirmatorios, revelaron una estructura de cuatro factores: urgencia, búsqueda de sensaciones, falta de premeditación y falta de perseverancia. El factor urgencia incluyó tanto la urgencia positiva como la urgencia negativa de la escala UPPS-P. La estructura factorial revelada se asemeja al UPPS, escala de impulsividad previa al UPPS-P, conformada por cuatro factores. Se conservó la totalidad de los ítems de la escala breve. Dos ítems presentaron mayor carga en un factor distinto al que pertenecían en la adaptación. Los valores del alfa de Cronbach fueron adecuados, lo cual aseguró la consistencia interna. El alfa de Cronbach de la escala total fue .79. La escala reveló validez convergente con los puntajes de psicopatía y del Inventario Experiencial Racional. Se observaron correlaciones parciales significativas entre maquiavelismo y narcisismo, y diferentes dimensiones de impulsividad, controlando psicopatía. Se demuestra que la escala de impulsividad en su versión breve mantiene adecuadas propiedades psicométricas en una muestra de adultos argentinos. Palabras clave: propiedades psicométricas, impulsividad, UPPS-P, Argentina. 


\section{Abstract}

This study aimed to explore the psychometric properties of the Impulsive Behavior Scale (UPPS-P) in its short version in a sample of Argentinean adults. Its factor structure and internal consistency were explored, as well as its convergent validity with experiential and rational thinking styles, along with psychopathy. At the same time, the relationship of impulsivity with narcissism and Machiavellianism was explored, controlling the scores in psychopathy. An intentional sample of Argentinean adults $(\mathrm{N}=517)$ between 18 and 60 years old (54\% female, mean age 27 ) was selected. Participants responded on-line to this scale, a sociodemographic inventory, as well as to the Rational Experiential Inventory and the Dark Personality Scale. The results obtained were processed with the programs SPSS 23, Factor 8.10, and AMOS 16.0. The sample was randomly divided into two groups of $n$ $=217$ and $\mathrm{n}=300$. An exploratory factorial analysis was first performed on the first group, as a calibration study. After that, a confirmatory analysis was performed on the second group, as a replication study, since there are no previous studies on this subject in Argentina. A parallel analysis was first carried out with the Factor 8.10 program. A number of replications $=100$ and percentile representation of simulations $=.95$ were used. Regarding the exploratory factor analysis, The Unweighted Least Squares method was used along with a Promin rotation. The final solution showed a structure of four factors: urgency, sensation seeking, lack of premeditation, and lack of perseverance, explaining $56 \%$ of the variance. Each of these factors explained $16 \%, 24 \%, 10 \%$, and $6 \%$ of the variance, respectively. The urgency factor included both the positive and negative urgency of the UPPS-P scale. The factor structure revealed is similar to the UPPS, a scale of impulsivity prior to the UPPS-P, made up of four factors. All items on the short scale were retained. Two items loaded better on a different factor. Cronbach's Alphas were adequate, ensuring internal consistency. Cronbach's $\alpha$ of the full scale was .79. Internal consistency was analyzed, resulting in Cronbach's $\alpha$ values of .76 for Emergency; .75 for Sensation Search; .69 for Lack of Premeditation, and .74 for Lack of Perseverance. The resulting Cronbach $\alpha$ values are similar to those of the Spanish version, which presented internal consistencies between .61 and .79. Subsequently, based on the factor structure found, confirmatory factor analysis was carried out on the second group sample. A relatively good fit was found for the model, with CFI and TLI values above .90 and the SRMR and RMSEA below .05. The authors' original model, which includes five factors, was also tested. The fit was less satisfactory compared to the previous model, because of the CFI, TLI, SRMR, and RMSEA indicators, and the difference in $\Delta$ from the models, which was statistically significant at the $\mathrm{p}<.001$ level. Finally, a second-order model was tested with all four dimensions as first-order latent variables and impulsivity as second-order latent variables. The fit was relatively satisfactory, with CFI and TLI above .90 and SRMR and RMSEA below .05. Thus, the present adjustment revealed a secondorder factor, as did the English short version of the UPPS-P (Cyders et al., 2014). The scale revealed convergent validity with the scores of psychopathy and the Rational Experiential Inventory. Psychopathy resulted positively related to impulsivity. In turn, the correlations between the dimensions of impulsivity and those of the Rational Experiential Inventory were negative with the rational thinking style -excluding sensation seeking- and positive with the significant ones of the experiential style. Significant partial correlations were observed between machiavellianism and narcissism and different dimensions of impulsivity, controlling psychopathy scores. It is demonstrated that the impulsivity scale in its short version maintains adequate psychometric properties in a sample of Argentinean adults.

Keywords: psychometric properties, impulsivity, UPPS-P, Argentina. 


\section{Introducción}

La impulsividad ha sido extensivamente incluida en modelos de personalidad, y se ha abordado como un constructo multidimensional, formado por rasgos que involucran la tendencia a decisiones rápidas, a actuar sin pensar y a involucrarse en conductas riesgosas (Cándido, Orduña, Perales, Verdejo-García y Billieux, 2012). La impulsividad se relaciona con diversas psicopatologías y demuestra su importancia en variadas formas de ellas, así como en distintos comportamientos problemáticos en población clínica y no clínica (Whiteside, Lynam, Miller y Reynolds, 2005), tales como personalidad antisocial y límite (Hahn, Simons y Hahn, 2016), alcoholismo (Whiteside y Lynam, 2003), dependencia de sustancias (Verdejo-García, Bechara, Recknor y Pérez-García, 2007), comportamientos sexuales de riesgo (Curry, Luk, Trim, Hopfer, Hewitt, Stallings y Wall, 2018), trastornos alimenticios (Claes, Vandereycken y Vertommen, 2005) y juego patológico (Savvidou, Fagundo, Fernández-Aranda, Granero, Claes, Mallorquí-Baqué y del Pino-Gutiérrez, 2017).

Whiteside y Lynam (2001) desarrollaron un modelo multidimensional de la impulsividad compuesto por cuatro dimensiones: la urgencia, la búsqueda de sensaciones, la falta de planificación y la falta de perseverancia. La urgencia es la incapacidad para controlar los impulsos en condiciones de afecto negativo, con elevada emocionalidad, e implica dificultad para resistir tentaciones y deseos fuertes (Whiteside et al., 2005). La búsqueda de sensaciones involucra la tendencia conductual hacia objetos y experiencias placenteras y emocionantes, así como la apertura a experiencias nuevas de este tipo, que pueden involucrar riesgos. La falta de planificación fue definida por los autores como la dificultad de proyectar consecuencias futuras de un acto antes de realizarlo, mientras que la falta de perseverancia implica la dificultad para mantener el foco en una tarea que puede ser aburrida, dificultosa o larga. Esto conlleva a dificultades para resistir estímulos distractores y a complicaciones para completar proyectos (Whiteside et al., 2005).

La Escala de Impulsividad UPPS original (Whiteside et al., 2005) presentaba las cuatro dimensiones anteriores. Para su desarrollo se utilizó una muestra de 122 participantes (90 $\%$ caucásicos) reclutados de salas de espera de un hospital, grupos de apoyo y centros de tratamiento. Se incluyeron individuos con trastorno límite de la personalidad, abuso de alcohol y jugadores patológicos, además de población comunitaria como control. Posteriormente, una quinta dimensión fue sumada al modelo, la urgencia positiva, que es definida como la tendencia a actuar de forma apresurada bajo afectos sumamente positivos (Cyders, Smith, Spillane, Fischer, Annus y Peterson, 2007). De este modo, la dimensión previamente denominada urgencia procedió a ser denominada urgencia negativa, lo que reveló una estructura factorial de cinco factores y dio origen al instrumento UPPS-P (Lynam, Smith, Whiteside y Cyders, 2006). Luego, este fue desarrollado en su versión corta en francés (Billieux, Rochat, Ceschi, Carré, Offerlin-Meyer, Defeldre y Van der Linden, 2012), en inglés (Cyders, Littlefield, Coffey y Karyadi, 2014), en portugués (Nogueira Sediyama, 2014), en italiano (D'Orta et al., 2015) y en alemán (Schmidt et al., 2008). También fue adaptado al español por Cándido et al. (2012) en su versión breve, para lo cual se utilizó una muestra de 189 participantes, de entre 18 y 45 años, que eran estudiantes universitarios con idioma español nativo o fluido.

El modelo propuesto por la UPPS-P presenta la ventaja de que las distintas facetas de la impulsividad se han asociado a una variedad de trastornos mentales (Cándido et al., 2012). Por otra parte, la UPPS-P presenta una versión larga y otra breve. Sin embargo, es importante destacar que existe un solapamiento conceptual e instrumental entre la impulsividad y la búsqueda de sensaciones (Steinberg, Albert, Cauffman, Banich, Graham y Woolard, 2008). La impulsividad 
se refiere a las deficiencias en la inhibición y falta de autocontrol que conllevan a comportamientos no planificados. La búsqueda de sensaciones, por su lado, implica la búsqueda de experiencias elevadamente estimulantes, variadas y novedosas, así como la voluntad de correr riesgos para obtenerlas (Zuckerman, 1979). La impulsividad y la búsqueda de sensaciones se encuentran moderadamente relacionadas (Zuckerman, 1979), a tal punto que componen un factor de personalidad llamado búsqueda de sensaciones impulsiva no socializada en una temprana investigación de rasgos de personalidad de Zuckerman, Kuhlman y Camac (1988). El Cuestionario de Personalidad de Zuckerman-Kuhlman (ZKPQ; Zuckerman, 2002) empleó análisis factoriales de escalas e ítems de distintos cuestionarios utilizados en estudios de temperamento o personalidad (Zuckerman, 2002). Una de estas cinco subescalas de esta escala más reciente persiste combinando la impulsividad y la búsqueda de sensaciones, y se denomina búsqueda de sensaciones impulsiva (ImpS) (Zuckerman, 2010). La forma final del ZKPQ consiste de 99 ítems verdadero-falso que se completan en 15 a 20 minutos. Una de sus subescalas es la búsqueda de sensaciones impulsiva (ImpSS), que fue dividida en un subfactor para impulsividad y en otro para la búsqueda de sensaciones (Zuckerman, 2008). Aquellos ítems de la subescala impulsividad describen la tendencia a actuar rápidamente sin pensar y la falta de planificación. Aquellos de búsqueda de sensaciones comprenden necesidad de novedad y cambio, excitación y elevada emoción, además de preferencia por situaciones impredecibles (Zuckerman, 2002). Además, Zuckerman planteó una búsqueda de sensaciones miltidimensional en su Escala de Búsqueda de Sensaciones (SSS; Zuckerman, Eysenck y Eysenck, 1978) en la que una de sus cuatro subescalas, denominada desinhibición, ya involucraba un componente de la impulsividad, que se observa en un menor control del comportamiento o desinhibición (Steinberg et al., 2008).
Sin embargo, según Steinberg y colaboradores (2008), la impulsividad y la búsqueda de sensaciones no implican el mismo constructo. Es importante considerar que no toda búsqueda de sensaciones es realizada de forma impulsiva, ni toda impulsividad conlleva a experiencias estimulantes (Steinberg et al., 2008). Si bien Zuckerman (2010) los combinó en un solo constructo, otro estudio demostró, mediante un análisis factorial confirmatorio, que un modelo de dos factores resultaba más apropiado que un unifactorial, lo cual implica que la impulsividad y la búsqueda de sensaciones representan constructos diferenciados (Magid, MacLean y Colder, 2007). Además, un estudio ha encontrado diferencias de edad en la búsqueda de sensaciones y en la impulsividad de individuos entre 10 y 30 años de edad. Por un lado, la búsqueda de sensaciones se incrementa entre los 10 y 15 años y disminuye o se mantiene estable luego, y está relacionada con la pubertad. En contraste, la impulsividad no se relaciona con la pubertad y presenta una caída lineal desde los 10 años en adelante (Steinberg et al., 2008). Según Verdejo-García y colaboradores (2010), la asociación de la búsqueda de sensaciones con las medidas de rasgos y estados de la impulsividad debería ser explorada más a fondo para mayor comprensión de sus roles diferenciados en el desarrollo de comportamientos desadaptativos.

La Impulsive Behavior Scale (UPPS-P), por su parte, incluye la búsqueda de sensaciones como un factor de la impulsividad (Whiteside y Lynam, 2001). Este factor de la UPPS-P fue compuesto a partir de escalas que evaluaban la búsqueda de aventura y excitación, entre las se incluían algunas como la desinhibición de la Sensation-Seeking-Scale (SSS; Zuckerman, Eysenck, y Eysenck, 1978), la impulsividad funcional de Dickman (DII; Dickman, 1990) y la faceta de búsqueda de excitación del inventario de personalidad NEO-PI-R (NEO-PI-R; Costa y McCrae,1992; Whiteside y Lynam, 2001).

Otro estudio ha desarrollado una escala de impulsividad dentro de un marco de perso- 
nalidad al igual que lo ha hecho Zuckerman (2002). Buss y Plomin (1975) incluyeron la impulsividad dentro de un modelo de cuatro factores del temperamento, junto a la emocionalidad, la actividad y la sociabilidad. Definieron la impulsividad como un temperamento con falta de control inhibitorio, poca habilidad de retrasar un comportamiento y de considerar consecuencias antes de tomar una decisión, tendencia a aburrirse y a buscar estímulos nuevos, y dificultad para mantenerse involucrado en una tarea frente a tentaciones (Whiteside y Lynam, 2001). Estos autores desarrollaron la escala de impulsividad Emotionality, Activity, Sociability, Impulsivity (EASI-III; Buss y Plomin, 1975) con 20 ítems ordenados en cuatro subescalas de impulsividad: control inhibitorio, tiempo de decisión, búsqueda de sensaciones y persistencia.

Otra escala que surgió relacionada a rasgos de personalidad como la extraversión, así como a la búsqueda de sensaciones, fue la Escala de Impulsividad de Barratt (BIS-11; Patton, Stanford y Barratt, 1995), conformada por tres subescalas: impulsividad atencional, impulsividad motora e impulsividad de falta de planificación. Esta escala fue implementada en poblaciones de estudiantes universitarios, población penitenciaria, pacientes con abuso de sustancias y pacientes psiquiátricos (Stanford, Mathias, Dougherty, Lake, Anderson, y Patton, 2009). Mediante un análisis de componentes principales, también se extrajeron seis factores de primer orden: atención, motricidad, autocontrol, complejidad cognitiva, perseverancia e inestabilidad cognitiva (Patton, Stanford y Barratt, 1995). Sin embargo, mientras que la escala BIS-11 desarrolla la impulsividad como un constructo multidimensional, Stanford y colaboradores (2009) expresan que la mayoría de los estudios que utilizan el BIS-11 ignoran las subescalas de primer y segundo orden, reportando únicamente el puntaje total. Otra escala que evalúa el constructo es la Escala de Impulsividad de Dickman (DII; Dickman, 1990). Sin embargo, dicha escala solo incluye dos dimensiones en su concepción de impulsividad: la impulsividad funcional y la impulsividad disfuncional (Dickman, 1990). La dimensión funcional implica la tendencia a la rápida toma de decisiones con riesgo calculado, significando un beneficio personal. La dimensión disfuncional, por su parte, incluye la toma de decisiones imprecisas y rápidas, sin reflexión, con consecuencias negativas (Pérez, 2009).

La UPPS-P presenta una solución a las numerosas conceptualizaciones y escalas de impulsividad, que resultaron en un uso inconsistente del constructo y producían resultados variados (Cyders et al., 2014). En este sentido, el modelo UPPS fue instaurado para resolver las limitaciones que implicaban la existencia de escalas de impulsividad que parecerían estar evaluando distintos constructos (Verdejo-García, Lozano, Moya, Alcázar y PérezGarcía, 2010). De esta forma, crea una especie de síntesis más actualizada, además de un punto de encuentro entre todas estas escalas de impulsividad comúnmente utilizadas, comprendiendo la impulsividad dentro del marco de un modelo general de la personalidad. Su versión breve constituye un ahorro de tiempo para un uso más pragmático en la práctica clínica e investigación. Además, la escala original UPPS demostró responder por un porcentaje considerable de la varianza en ciertas psicopatologías, particularmente para el trastorno de personalidad límite y el antisocial (Whiteside et al., 2005)

La relación entre la tríada de personalidad oscura y la impulsividad ha sido explorada previamente (Jones y Paulhus, 2011). La tríada de personalidad oscura es un constructo elaborado por Paulhus y Williams (2002) que reúne tres tipos de personalidades malévolas socialmente las cuales presentan cualidades como baja amabilidad, deshonestidad, frialdad emocional, agresividad y tendencias hacia la autopromoción (Paulhus y Williams, 2002). Dichas personalidades son el maquiavelismo, el narcisismo y la psicopatía. Es importante reconocer que se trata de personalidades 
socialmente aversivas, pero no patológicas (Furnham, Richards y Paulhus, 2013).

La relación entre las distintas personalidades oscuras y la impulsividad fue explorada en un estudio de Jones y Paulhus (2011) en el cual el maquiavelismo no presentó relación con la impulsividad. El narcisismo, por su parte, demostró relacionarse con la impulsividad funcional, mientras que la psicopatía se vinculó con la impulsividad disfuncional. Por otro lado, un estudio reveló la impulsividad únicamente en las personalidades narcisista y psicópata (Malesza y Ostaszewski, 2016) y otro la relacionó también con el maquiavelismo (Crysel, Crosier y Webster, 2013). También se destaca la importancia del valor explicativo de la impulsividad sobre ciertos comportamientos de los miembros de la tríada oscura. La conducta de autosabotaje de los narcisistas, consistentes en su comportamiento hostil en las interacciones sociales, discordante con el feedback positivo que desean, fue explicada por Vazire y Funder (2006) como falta de autocontrol y preferencia por beneficios a corto plazo. Por otra parte, la estrategia de explotación de las personalidades oscuras es alimentada por una estrategia de vida rápida, tendencia a no pensar en posibles consecuencias y falta de autocontrol (Jonason y Tost, 2010).

Dado que un factor de la impulsividad, según el modelo UPPS-P, es la falta de planificación, resulta interesante involucrar en este estudio una variable que evalúe el pensamiento en relación a la impulsividad. Pacini y Epstein (1999) plantean que existen dos modos paralelos de procesar la información: el modo racional y el modo experiencial. Dichos modos implican dos tipos de pensamiento distintos. El pensamiento experiencial es influenciado emocionalmente, es asociativo, automático y permite solucionar problemas rápidamente. Además, se encuentra relacionado con la intuición e imaginación. El pensamiento racional, por su parte, soluciona problemas de forma lógica; es analítico, consciente, lento $\mathrm{y}$ se relaciona con el autocontrol (Norris y
Epstein, 2011; Pacini y Epstein, 1999). Si bien ambos modos operan en conjunto, los individuos suelen inclinarse a utilizar un estilo de pensamiento de forma predominante (Riding, Rayner y Rayner, 2000).

Un estudio reveló, indirectamente, ciertas relaciones entre la impulsividad y el estilo de pensamiento. La urgencia, más allá de su carácter positivo o negativo -incapacidad de controlar los impulsos bajo emociones intensas positivas o negativas-, tiende a disminuir la toma de decisiones racional, resultado parcialmente explicado por una prioridad del foco en necesidades a corto plazo (Cyders y Smith, 2008). En relación con la personalidad oscura, los individuos maquiavélicos resultan ser fríos, racionales y estratégicos en la toma de decisiones, y las realizan de manera impersonal y con desapego afectivo (Czibor, Vincze y Bereczkei, 2014). Sin embargo, no se han llevado a cabo investigaciones que empleen el instrumento de estilo de pensamiento Rational Experiental Inventory (REI; Pacini y Epstein, 1999) en relación con la impulsividad o personalidad oscura. Un estudio reciente en juicio moral ha demostrado que la tríada de personalidad oscura se relaciona positivamente con inclinaciones utilitaristas (Djeriouat y Trémolière, 2014). Además, emergiendo de un procesamiento más frío y deliberado, los juicios utilitarios -relacionados con dichas personalidades - resultan ser predichos por el estilo de pensamiento racional (Bartels, 2008).

Resulta relevante la adaptación de instrumentos de apropiadas propiedades psicométricas para la evaluación de la impulsividad y sus distintas dimensiones, aunque, si bien han cumplido con tales requisitos, poseen sus limitaciones. La adaptación de la UPPS-P al español en su versión breve (Cándido et al., 2012) y al francés (Billieux et al., 2012) fueron desarrolladas empleando muestras de estudiantes universitarios (Verdejo-García et al., 2010). En este sentido, resulta acorde a estas limitaciones examinar las propiedades psicométricas de dicha escala en una muestra conformada por adultos que no sean estudi- 
antes universitarios y de un rango etario más amplio. Por otra parte, si bien ya existe una versión adaptada al español de esta escala, la versión breve ha sido desarrollada sobre una muestra de España. Además, otra limitación de la escala adaptada al español de la UPPS-P implica el desbalance de sexos de la muestra -el $92 \%$ estaba compuesto por mujeres(Verdejo-García et al., 2010). De esta forma, se rescata la importancia de evaluar las propiedades psicométricas de dicha escala en una muestra argentina, examinando su validez intercultural, más balanceada según los sexos. A su vez, se ha destacado la necesidad de aportar evidencia de validez de constructo mediante la inclusión de correlaciones con otros constructos teóricamente relacionados con la impulsividad (Verdejo-García et al., 2010). Por otra parte, la ventaja de dicha escala es que presenta una versión breve de 20 preguntas y, además, es un instrumento ampliamente usado a nivel mundial y traducido a numerosas lenguas.

Teniendo en cuenta los antecedentes mencionados, la presente investigación tenía como objetivos:

a) explorar la estructura factorial de la Escala de Impulsividad (UPPS-P) en su versión breve y su consistencia interna en una muestra de adultos argentinos;

b) examinar su validez convergente con el estilo de pensamiento experiencial, el estilo de pensamiento racional y con la psicopatía.

c) determinar las relaciones de la impulsividad con el narcisismo y el maquiavelismo, controlando la psicopatía.

\section{Método}

\section{Participantes}

La muestra intencional no probabilística consistió en 517 adultos argentinos de ambos sexos (54\% femenino y $64 \%$ masculino; edad promedio: 27 años, $D E=10.55$ ) de edades comprendidas entre 18 y 60 años, nacidos en la Provincia de Tierra del Fuego, Antártida e Islas del Atlántico Sur (30.4\%), la Ciudad Autónoma de Buenos Aires (36.6\%) y la Provincia de Buenos Aires (33.1\%), todos reclutados mediante diversas redes sociales.

Los criterios de inclusión fueron los siguientes: (a) hombres y mujeres de población general, de cualquier estado civil y nivel de estudios, que supieran leer y utilizar computadora o celular inteligente, que tuvieran acceso a la encuesta online y aceptaran responderla en su totalidad; (b) de una edad entre $18 \mathrm{y}$ 60 años; y (c) nacidos en Tierra del Fuego, Provincia de Buenos Aires, o Ciudad Autónoma de Buenos Aires. Por otra parte, se excluyeron participantes nacidos en otras localidades, personas que no supieran leer y escribir, que no manejen celulares o computadoras, menores de edad y mayores de 60 años.

\section{Instrumentos}

\section{UPPS-P Impulsive Behavior Scale (UPPS-P; Lynam, Smith, Whiteside y Cyders, 2006)}

Esta escala fue adaptada al español en versión breve por Cándido, Orduña, Perales, Verdejo-García y Billieux (2012) y está constituida por 20 de los 59 ítems de la escala original, agrupados en cinco dimensiones (cuatro ítems por dimensión).

Partiendo de una concepción multidimensional, evalúa la impulsividad desde cinco factores que pueden conducir a comportamientos impulsivos: urgencia negativa (tendencia a actuar impulsivamente cuando se experimenta una situación negativa), urgencia positiva (comportamiento impulsivo al experimentar una situación positiva), búsqueda de sensaciones (búsqueda de actividades excitantes y preferencia conductual por objetos placenteros), falta de premeditación (tendencia a actuar sin pensar en las consecuencias) y falta de perseverancia (incapacidad para finalizar una tarea) (Whiteside y Lynam, 2001). Se trata de un cuestionario 
de autoevaluación cuyos ítems se basan en la descripción de rasgos y comportamientos impulsivos, a los que el participante responde según el grado en que se apliquen a sí mismo. El cuestionario consiste en una escala Likert de cuatro opciones de grado de acuerdo, desde 1 (Totalmente en desacuerdo) hasta 4 (Totalmente de acuerdo) (Lynam et al., 2006). En la versión española, las opciones de grado de acuerdo también son cuatro, desde 1 (Rotundamente de acuerdo) hasta 4 (Rotundamente en desacuerdo). Ejemplos de los ítems son: "Cuando estoy irritado suelo actuar sin pensar"; "Generalmente busco experiencias y sensaciones nuevas y excitantes"; "Casi siempre termino los proyectos que empiezo"; "Cuando estoy realmente animado, no suelo pensar en las consecuencias de mis acciones", y "Mi manera de pensar es normalmente meticulosa y centrada" (Cándido et al., 2012).

La adaptación breve en español utilizada en el presente estudio (Cándido et al., 2012) se basó en la adaptación breve francesa de Billieux y colaboradores (2012) y presenta cuatro ítems por dimensión, los cuales fueron seleccionados por presentar mayores cargas factoriales en cada dimensión. La consistencia interna fue satisfactoria, con un alfa de Cronbach de entre .70 y .84 entre dimensiones, y correlaciones test-retest de entre .84 y .82 . Los análisis factoriales confirmatorios demostraron una estructura de cinco dimensiones interrelacionadas, así como otro modelo con una estructura con dos factores de segundo orden: urgencia -integrada por la urgencia negativa y la urgencia positiva-, y falta de consciencia -que reúne la falta de premeditación y la falta de perseverancia. Por su parte, la búsqueda de sensaciones constituyó un factor diferenciado (Billieux et al., 2012). En la versión española de Cándido y colaboradores (2012) los valores de alfa de Cronbach oscilaron entre .61 y .81 entre dimensiones, con una estructura factorial que coincidió con el modelo de cinco factores. Sin embargo, los factores de segundo orden de la adaptación francesa (Billieux et al., 2012) no fueron hallados en esta adaptación. De esta forma, la versión breve de la UPPS-P adaptada al español a partir de una muestra de estudiantes universitarios demostró adecuada estructura factorial, correcta consistencia interna y validez de constructo (Cándido et al., 2012). Previamente, dos jueces independientes expertos en psicología controlaron la equivalencia entre los términos usados e indicaron cambios menores $y$, antes de utilizarla para la presente investigación, se la aplicó a una muestra piloto de 52 alumnos universitarios. No surgieron dudas al responder y los valores de alfa de Cronbach resultaron adecuados.

\section{Short Dark Triad (SD3; Jones y Paulhus, 2014)}

Este instrumento fue adaptado al castellano por Nohales Nieto (2015) y evalúa la personalidad oscura de forma subclínica en tres dimensiones: maquiavelismo, narcisismo y psicopatía. Su versión original se aplicó a una muestra de 245 estudiantes universitarios. Los valores de alfa de Cronbach para las subescalas fueron de .71 para narcisismo, .77 para maquiavelismo y .80 para psicopatía. También se comprobó que cada subescala resultó ser un proxy para cada uno de los instrumentos que evaluaban personalidades oscuras que buscaban reemplazar. Las tres subescalas representaron en su totalidad los constructos clásicos (Jones y Paulhus, 2014). Por su parte, la adaptación al español se desarrolló a partir de una muestra de 243 participantes de entre 18 y 63 años y su consistencia interna fue adecuada, con alfas de Cronbach similares a los de la escala original: .697 para narcisismo y mayor a .7 para maquiavelismo y psicopatía. También se reveló fiabilidad test-retest y se indicaron correlaciones altas entre las dimensiones (Nohales Nieto, 2015).

Se trata de un cuestionario autoadministrado que en su versión original fue integrado por 27 ítems, con nueve ítems por subescala que evalúan diferentes facetas del constructo -maquiavelismo, narcisismo y psicopatía. Sin 
embargo, en la adaptación de Nohales Nieto (2015) se eliminaron los ítems 21, 27 y 29 debido a su baja saturación. Además, el ítem 14 "Me gusta usar argucias para conseguir lo que quiero", que integraba la subescala de maquiavelismo, fue incluido en la de psicopatía por su mejor saturación con este factor. De esta forma, la presente adaptación resultó constituida por 24 ítems en tres subescalas, correspondientes a cada una de las personalidades oscuras. Las dimensiones maquiavelismo y narcisismo contienen siete ítems cada una, mientras que la dimensión psicopatía es integrada por 10 ítems. Las alternativas de respuesta del cuestionario son constituidas por una escala Likert integrada por cinco opciones de grado de acuerdo, desde 1 (Totalmente en desacuerdo) hasta 5 (Totalmente de acuerdo). Ejemplos de los ítems de la subescala de psicopatía son: "Es cierto que puedo ser cruel con los demás" y "Me gusta vengarme de las figuras de autoridad". Dentro de los ítems de la subescala de maquiavelismo encontramos "La mayoría de la gente puede ser manipulada" y "Evito el conflicto directo con otros porque me pueden ser de utilidad en el futuro". Algunos ejemplos de la subescala de narcisismo son: "La gente me ve como un líder natural" y "Muchas actividades en grupo tienden a ser aburridas sin mí".

\section{Rational Experiential Inventory (REI; Pacini y Epstein, 1999)}

Fue adaptado al castellano por Reyna y Ortiz (2016) y evalúa la preferencia por el estilo de pensamiento racional o experiencial. En su versión original se utilizó una muestra de estudiantes universitarios con una edad promedio de 20 años. Su adaptación al castellano también se realizó con una muestra de estudiantes universitarios con 395 participantes, en su mayoría de la Universidad Nacional de Córdoba (Argentina). Este cuestionario autoadministrado evalúa aquel estilo de pensamiento predominante en el participante mediante ítems que contienen una variedad de variables de personalidad, estilo de pensamiento y creencias ideológicas relevantes para los modelos racional y experiencial. El instrumento se diferencia de otros instrumentos que evalúan dos maneras similares de procesamiento de información, al ser el único que los sitúa en un contexto de una teoría global de la personalidad y evalúa las diferencias individuales por las que una persona opera predominantemente con el modelo experiencial o el racional. Se encuentra integrado por 40 ítems y dos dimensiones. La dimensión racional, compuesta por 20 ítems, está constituida por dos subescalas: la habilidad racional -habilidad para pensar de manera analítica- y el compromiso racional-disfrute al pensar de forma racional. La dimensión experiencial, también integrada por 20 ítems, se encuentra compuesta por la habilidad experiencial -habilidad de identificar intuiciones- y el compromiso experiencial -disfrute al tomar decisiones basadas en su intuición (10 ítems). En cuanto a la consistencia interna del instrumento original, es destacable que la escala racional obtuvo un alfa de Cronbach de .90, mientras que la escala experiencial obtuvo un alfa de .87. La correlación entre ambas escalas no fue significativa, lo que confirmó la independencia de estos dos modos de procesamiento de información. Análisis de regresiones demostraron que ambas escalas implican contribuciones únicas en la predicción de variables que representan dimensiones básicas de la personalidad. Las subescalas de habilidad y compromiso también fueron apoyadas, demostrando validez discriminante. Su versión adaptada al castellano (Reyna y Ortiz, 2016) fue validada en la Argentina y presentó consistencia interna satisfactoria, con un alfa de Cronbach de .855 para el factor experiencial y de .814 para el factor racional. Los análisis factoriales exploratorios y confirmatorios revelaron la misma estructura de dos factores que la escala original. Las opciones de respuesta se constituyen como una escala Likert que consiste en cinco opciones de grado de acuerdo -de total- 
mente en desacuerdo a totalmente de acuerdo. Ejemplos de los ítems son "Prefiero los problemas complejos antes que los simples"; "No soy una persona que piense de manera muy analítica"; "No me gustan las situaciones en las cuales tengo que confiar en mi intuición"; "Soy mucho mejor que la mayoría de la gente para resolver las cosas de forma lógica", y "No razono bien bajo presión".

\section{Procedimiento de recolección de datos}

Los participantes fueron reclutados mediante diversos medios de comunicación virtual y los datos fueron recolectados mediante una batería en formato online, a través de la plataforma Google Forms mediante redes sociales como Facebook e Instagram. Se utilizaron escalas de autoevaluación para la valoración de cada una de las variables incluidas en el modelo. Cada participante respondió a los cuestionarios en una sola sesión a través de la plataforma mencionada, con la inclusión de un consentimiento informado, lo cual aseguró el anonimato y confidencialidad de las respuestas y el cumplimiento de los requisitos por parte de los participantes. La investigación fue aprobada por la Universidad Argentina de la Empresa, CABA, Argentina.

\section{Procedimiento de análisis de datos}

En primer lugar, se dividió aleatoriamente la muestra en dos grupos de $\mathrm{n}=217$ y $\mathrm{n}=$ 300. Con el primero se realizó un análisis paralelo con el programa Factor 8.10 y con el otro un análisis factorial confirmatorio con el programa AMOS 17.0. Los datos se procesaron en el programa SPSS 23 para llevar a cabo correlaciones de Pearson con el instrumento racional experiencial, con el objetivo de evaluar la validez convergente del instrumento. A su vez, se realizaron correlaciones parciales para explorar las relaciones de la impulsividad con el narcisismo y el maquiavelismo, controlando la psicopatía. Dicha nece- sidad se debió a que, como la relación entre la impulsividad y la psicopatía ha sido sustentada teóricamente, resultó relevante explorar las relaciones con las otras dos personalidades oscuras controlando la psicopatía. De esta forma, evitamos una relación espuria, que las relaciones de la psicopatía con el narcisismo y el maquiavelismo fueran las responsables de las relaciones que demuestre la impulsividad con estos dos últimos constructos.

Como se deseaba explorar los factores que emergerían de la escala, primero se procedió a llevar a cabo un análisis paralelo con el programa Factor 8.10 (Lorenzo-Seva y Ferrando, 2013). Se empleó un número de replicaciones igual a .100 y percentil de representación de simulaciones de .95 . Se empleó el método de implementación clásico de Horn (1965) para determinar la retención de factores. Para la retención, se compararon los autovalores empíricos con los aleatorios (medias), seleccionando aquellos que se encontraban por encima de la media aleatoria (O'Connor, 2000). Con respecto al análisis factorial exploratorio, se usó rotación Promin debido a que se postulaba que los factores estarían asociados. Asimismo, dicha rotación permite que los factores sean oblicuos, facilitando la simplificación de la estructura factorial (Lorenzo-Seva, 2013). Se usó el método Unweighted Least Squares para dicho análisis, debido a la naturaleza ordinal de los datos y a que las opciones de respuestas eran de cuatro alternativas.

Para el Análisis Factorial Confirmatorio (AFC) y evaluar su ajuste, se tuvieron en cuenta el Índice de Ajuste Comparativo (CFI) y el Î́ndice de Tucker-Lewis (TLI), los cuales deben tener valores por encima de .90; como también el error de aproximación cuadrático medio (RMSEA) y el residuo estandarizado cuadrático medio (SRMR), los cuales deben presentar valores menores de .10 (Bentler, 1992; Byrne, 2010). También se tuvieron en cuenta criterios más exigentes de CFI y TLI mayores de .95 y RMSEA y SRMR menores de .05 (Hu y Bentler, 1999). Que el chi-cua- 
drado $\left(\chi^{2}\right)$ no sea significativo es un criterio muy exigente y sensible al tamaño de la muestra (Byrne, 2010), por lo cual se dividió el $\chi^{2}$ por los grados de libertad del modelo. Aunque no hay criterios unánimes del valor, se establece generalmente que debe ser menor a tres. Se puso a prueba un modelo confirmatorio y, posteriormente, un modelo de segundo orden. Se empleó el modelo Maximum likelihood (ML), tanto para el análisis factorial exploratorio (AFE) como para el AFC, debido a que los valores tenían una distribución normal: la asimetría iba de .054 a 1.001 y la curtosis iba de .156 a .878 , ya que valores de 3 y 8 son considerados extremos (Boomsma y Hoogland, 2001; Byrne, 1989; Weston y Gore, 2006). Aunque el formato de respuestas es ordinal, como en muchos otros tests, su distribución normal amerita que se trate a los datos de forma continua (Schmidt et al., 2008). Se comenzó por realizar un análisis factorial exploratorio (un estudio de calibración), para luego realizar un análisis confirmatorio (un estudio de replicación) debido a que no existen estudios a este respecto en la Argentina. Se optó por este enfoque basados en los datos o botton up -primero un exploratorio y luego un confirmatorio- porque se sabe que las estructuras factoriales de un instrumento pueden variar de un estudio a otro o cuando se está en un proceso de adaptación de un test (Fehm y Hoyer, 2004; Wells y Davies, 1994).

\section{Resultados}

Primeramente, se realizó un análisis paralelo con el fin de examinar la estructura factorial de la Escala de Impulsividad (UPPS-P) con la muestra de $n=217$. La prueba de esfericidad de Bartlett fue significativa $\chi^{2}(190)=$ $3860.10, \mathrm{p}<.0001 \mathrm{y}$ el indicador de adecuación del tamaño de muestra Kaiser-Meyer-
Olkin fue adecuado (.801) para llevarlo a cabo. Al comparar los autovalores empíricos $\mathrm{y}$ aleatorios, los primeros cuatro autovalores empíricos se hallaban por encima de la media aleatoria, por lo cual se decidió retener cuatro factores, como se sugiere con este procedimiento (O’Connor, 2000).

Con respecto al análisis factorial exploratorio, al retener cuatro factores, se explicaba un $56 \%$ de la varianza. Cada uno de ellos explicaba un $16 \%, 24 \%, 10 \%$ y $6 \%$ de la varianza, respectivamente. Los resultados del análisis factorial se presentan en la Tabla 1. Como se puede observar en dicha tabla, no se replicaba la estructura de cinco factores postulada por los autores. En cambio, emergía una de cuatro factores y no existían cargas cruzadas superiores a .30. El primer factor se denominó Urgencia y se compuso de los ítems 2, 4, 7, 12, 15, 17 y 20 de la escala. El segundo factor, llamado Búsqueda de Sensaciones, se compuso de los ítems 3, 9, 10, 14 y 18. El tercer factor se denominó Falta de Premeditación y fue integrado por los ítems 1, 5, 6, 13 y 19. El cuarto factor, llamado Falta de Perseverancia, se compuso por los ítems 8, 11 y 16 de la escala.

De esta forma, los ítems de urgencia negativa y urgencia positiva constituyeron un solo factor de urgencia, mientras que los demás factores mantuvieron su estructura, a excepción de dos ítems. El ítem 10 "Cuando estoy rebosante de alegría, siento que no puedo evitar tirar la casa por la ventana", que correspondía a urgencia positiva, presentó mayor carga en el factor de búsqueda de sensaciones. El ítem 5 "En general me gusta asegurarme de llevar las cosas a buen término", que pertenecía al factor de falta de perseverancia, presentó mayor carga en el factor de falta de premeditación. 


\section{Tabla 1}

Análisis factorial exploratorio para los items de la escala de impulsividad (UPPS-P) breve en una muestra de adultos argentinos.

\begin{tabular}{|c|c|c|c|c|}
\hline $\begin{array}{c}\text { Ítems } \\
\text { Impulsividad } \\
\end{array}$ & Factor 1 & Factor 2 & Factor 3 & Factor 4 \\
\hline 1 & & & .727 & \\
\hline 2 & .449 & & & \\
\hline 3 & & .669 & & \\
\hline 4 & .824 & & & \\
\hline 5 & & & .446 & \\
\hline 6 & & & .695 & \\
\hline 7 & .702 & & & \\
\hline 8 & & & & .941 \\
\hline 9 & & .676 & & \\
\hline 10 & & .332 & & \\
\hline 11 & & & & .790 \\
\hline 12 & .721 & & & \\
\hline 13 & & & .879 & \\
\hline 14 & & .669 & & \\
\hline 15 & .397 & & & \\
\hline 16 & & & & .503 \\
\hline 17 & .606 & & & \\
\hline 18 & & .874 & & \\
\hline 19 & & & .417 & \\
\hline 20 & .396 & & & \\
\hline $\begin{array}{c}\% \text { de varianza } \\
\text { explicada }\end{array}$ & 16 & 24 & 10 & 6 \\
\hline
\end{tabular}

Nota. Se omiten las cargas factoriales menores a 30 .

Posteriormente, se realizó un análisis factorial confirmatorio a partir de la estructura factorial encontrada en el análisis paralelo y el análisis factorial exploratorio con la muestra de $n=300$. El modelo a poner a prueba se expone en la Figura 1. Se encontró un ajuste relativamente adecuado para el modelo, como se muestra en la Tabla 2, ya que el CFI y el TLI se hallaban por encima de .90 y el SRMR y RMSEA se encontraba por debajo de .05. Aunque el $\chi^{2}$ era significa- tivo, al dividirlo por los grados de libertad se hallaba por debajo de tres, lo cual es indicio de un buen ajuste (Cupani, 2012). También se puso a prueba el modelo original de los autores de cinco factores; como se muestra en la Tabla 2, el ajuste era menos satisfactorio, no solo por los indicadores de CFI, TLI, SRMR y RMSEA, sino porque la diferencia de $\Delta \chi$ de los modelos era estadísticamente significativa a nivel $p<.001$. Finalmente, se puso a prueba un modelo de segundo orden con las cuatro 
dimensiones como variables latentes de primer orden y la impulsividad como variable latente de segundo orden. En la Tabla 2 se muestra el ajuste de dicho modelo el cual era relativamente satisfactorio, ya que el CFI y el TLI se hallaban por encima de $.90 \mathrm{y}$ el SRMR y RMSEA se encontraba por debajo de .05. A su vez, se analizaron las consistencias internas y resultaron valores $\alpha$ de Cronbach de .76 para Urgencia, .75 para Búsqueda de Sensaciones, .69 para Falta de Premeditación, y de .74 para Falta de Perseverancia. El $\alpha$ de Cronbach de la escala total fue .79 . 


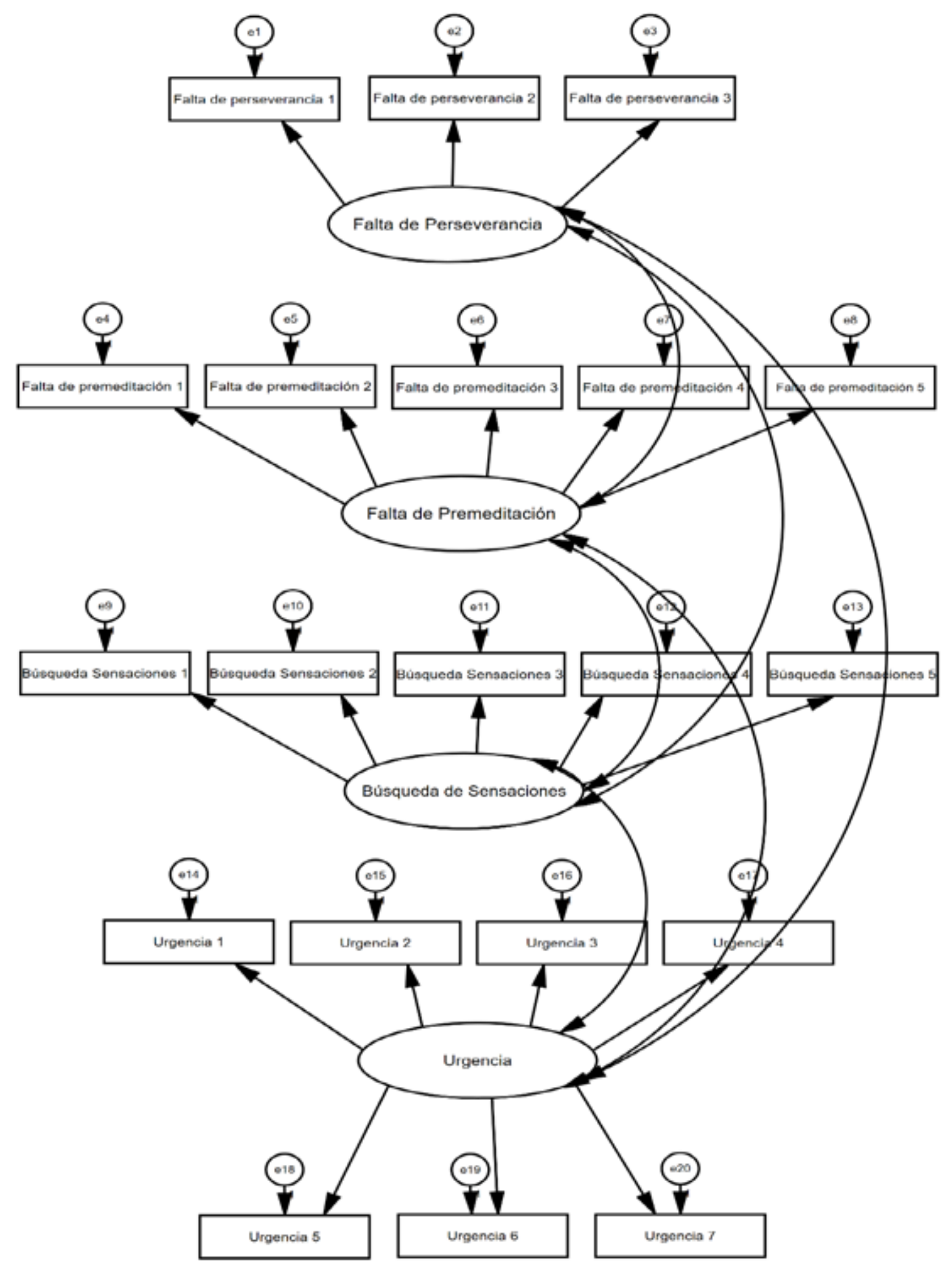

Figura 1. Modelo factorial confirmatorio de la escala de impulsividad breve UPPS-P de Lynam et al. (2006) de cuatro factores: urgencia, búsqueda de sensaciones, falta de premeditación y falta de perseverancia. 
Tabla 2

Índices de ajuste de los modelos de la escala de impulsividad (UPPS-P) breve en una muestra de adultos argentinos.

\begin{tabular}{c|c|c|c|c|c|c|c|c|c}
$\begin{array}{c}\text { Estadísticos de } \\
\text { ajuste }\end{array}$ & $\boldsymbol{\chi}^{\mathbf{2}(\mathbf{g l})}$ & $\mathbf{p}$ & $\mathbf{S R M R}$ & $\mathbf{R M S E A}$ & $\mathbf{T L I}$ & $\mathbf{C F I}$ & $\Delta \boldsymbol{\chi} \mathbf{2}$ & $\Delta \mathbf{g l}$ & $\Delta \mathbf{C F I}$ \\
\hline $\begin{array}{c}\text { Modelo de cuatro } \\
\text { factores }\end{array}$ & $367.112(164)$ & .001 & .036 & .048 & .913 & .925 & & & \\
$\begin{array}{c}\text { Modelo de cinco } \\
\text { factores }\end{array}$ & $401.478(160)$ & .001 & .040 & .062 & .881 & .902 & 34.366 & 4 & .023 \\
$\begin{array}{c}\text { Modelo de } \\
\text { segundo orden }\end{array}$ & $363.003(166)$ & .001 & .039 & .049 & .911 & .923 & & &
\end{tabular}

Para analizar la validez convergente, se llevaron a cabo correlaciones de Pearson entre las dimensiones de la impulsividad y ambos factores del Inventario Racional Experiencial, como también entre la impulsividad y la psicopatía.

Se hallaron correlaciones significativas de forma esperada entre todas las subescalas de impulsividad y los factores racionales y experienciales. La única excepción fue la falta de asociaciones significativas entre falta de perseverancia y estilo experiencial, como se puede observar en la tabla 3. Las asociaciones resultaron ser positivas entre el estilo experiencial y las restantes tres dimensiones de la impulsividad. El estilo racional presentó una correlación positiva únicamente con la búsqueda de sensaciones, y mostró asociaciones negativas con todas las restantes dimensiones de impulsividad. Según dichas relaciones, a mayor puntaje en impulsividad, excepto en la búsqueda de sensaciones, menor puntaje en estilo racional.

Al explorar las correlaciones entre impulsividad y psicopatía, se encontró que se correlacionó de forma significativa y positiva con todas las dimensiones de la impulsividad. Las asociaciones más fuertes fueron las que se correspondían con urgencia y búsqueda de sensaciones.

Tabla 3

Correlaciones entre impulsividad, personalidad oscura, y estilo de pensamiento.

\begin{tabular}{c|c|c|c|c} 
& Urgencia & $\begin{array}{c}\text { Falta de } \\
\text { premeditación }\end{array}$ & $\begin{array}{c}\text { Falta de } \\
\text { perseverancia }\end{array}$ & $\begin{array}{c}\text { Búsqueda de } \\
\text { sensaciones }\end{array}$ \\
\hline Estilo racional & $-.304 * *$ & $-.426^{* *}$ & $-.400^{* *}$ & $.169 * *$ \\
Estilo experiencial & $.234 * *$ & $.130 * *$ & -.049 & $.200 * *$ \\
Psicopatía & $.335^{* *}$ & $.190 * *$ & $.189 * *$ & $.361 * *$
\end{tabular}

${ }^{*} \mathrm{p}<.05 ; * * \mathrm{p}<.01$.

Para la resolución del objetivo que consistió en explorar las relaciones entre la impulsividad, el maquiavelismo y el narcisismo, controlando los puntajes en psicopatía, se llevaron a cabo correlaciones parciales. Los resultados se observan en la tabla 4 .
El maquiavelismo se correlacionó de forma significativa y negativa con falta de premeditación. Por su parte, el narcisismo se correlacionó de forma significativa y positiva con búsqueda de sensaciones y de forma negativa con falta de perseverancia. 
Tabla 4

Correlaciones parciales entre impulsividad maquiavelismo y narcisismo, controlando psicopatía.

\begin{tabular}{c|c|c|c|c} 
& Urgencia & $\begin{array}{c}\text { Falta de } \\
\text { premeditación }\end{array}$ & $\begin{array}{c}\text { Falta de } \\
\text { perseverancia }\end{array}$ & $\begin{array}{c}\text { Búsqueda de } \\
\text { sensaciones }\end{array}$ \\
\hline Maquiavelismo & $.086^{*}$ & $-.184^{* *}$ & -.017 & .036 \\
Narcisismo & .063 & -.068 & $-.258^{* *}$ & $.229 * *$
\end{tabular}

$* \mathrm{p}<.05 ; * * \mathrm{p}<.01$

\section{Discusión}

La UPPS-P en su versión breve nunca había sido adaptada a la Argentina. Entre las ventajas del presente estudio está el trabajar con una muestra de diversas regiones del país. Además, los resultados se detectaron con una medición multidimensional de la impulsividad y se controlaron los puntajes de la psicopatía al tratarse de las correlaciones. Otra ventaja es que dicha escala es una de las más utilizadas para medir la impulsividad a nivel multidimensional. A su vez, mostró buenas propiedades psicométricas y fue adaptada a numerosas lenguas y países.

La impulsividad es un constructo multidimensional que resulta de vital importancia para la caracterización de comportamientos de riesgo y de desórdenes psicopatológicos que involucran disfunciones en el control de los impulsos. Si bien existen instrumentos con satisfactorias propiedades psicométricas para evaluarlo, el uso inconsistente de este término y la posibilidad de que estas escalas estuviesen evaluando constructos diferentes han interferido en la evaluación de dicho constructo. El instrumento UPPS-P, que ha respondido a dichas limitaciones, también ha demostrado sólidas propiedades psicométricas en su versión original y en sus versiones cortas en francés (Billieux et al., 2012), en inglés (Cyders et al., 2014) y en español (Cándido et al., 2012). Sin embargo, esta última ha demostrado dichas propiedades en una muestra de estudiantes universitarios $\mathrm{y}$, en el presente estudio, se revelaron por primera vez en una muestra argentina.

Los análisis exploratorios y confirmatorios revelaron cuatro factores para la escala: urgencia, búsqueda de sensaciones, falta de premeditación y falta de perseverancia. El modelo presentó índices de ajustes satisfactorios más adecuados con esta estructura de cuatro factores, en comparación con aquella de las adaptaciones breves ya existentes en francés, inglés y en español, de cinco factores, que diferencian la urgencia negativa de la urgencia positiva. En este sentido, la estructura factorial coincide con aquella del instrumento UPPS original en inglés (Whiteside et al., 2005) que reveló cuatro factores: urgencia, falta de premeditación, falta de perseverancia y búsqueda de sensaciones. Sin embargo, presenta los mismos ítems que la adaptación española por Cándido et al. (2012). A pesar de ello, el ítem 10 "Cuando estoy rebosante de alegría, siento que no puedo evitar tirar la casa por la ventana", que correspondía a urgencia positiva, ahora forma parte de búsqueda de sensaciones. El ítem 5 "En general me gusta asegurarme de llevar las cosas a buen término", que pertenecía al factor de falta de perseverancia, ahora pertenece al factor de falta de premeditación. Por su parte, la presente adaptación no reveló un mejor ajuste con un factor de segundo orden, a diferencia de la versión breve en inglés de la UPPS-P (Cyders et al., 2014). Por otro lado, se obtenía un porcentaje satisfactorio de explicación de la varianza que superaba un $56 \%$, aunque dos factores explicaban un porcentaje menor de $10 \%$ y $6 \%$, pero el análisis paralelo indicó retener cuatro factores y el AFC mostró un ajuste adecuado. 
Con respecto a la consistencia interna del instrumento, las $\alpha$ de Cronbach resultaron adecuadas, con valores de entre .69 y .76 en sus subescalas, y un $\alpha$ de Cronbach de la escala total de .79. Sin embargo, en comparación con adaptaciones en otros idiomas, las del presente estudio resultan ligeramente satisfactorios. La adaptación breve en inglés de Cyders y colaboradores (2014) mostró $\alpha$ de Cronbach entre .74 y .85. Por su parte, la adaptación francesa de Billieux y colaboradores (2012) reveló valores de $\alpha$ de Cronbach entre .70 y .84. Sin embargo, los valores de $\alpha$ de Cronbach resultantes se asemejan a los de la versión española de Cándido y colaboradores (2012), que presentó consistencias de entre .61 y .79 .

Las correlaciones entre las subescalas de impulsividad y las del Inventario Racional Experiencial también hallaron los resultados esperados: fueron negativas con el estilo de pensamiento racional -excluyendo la búsqueda de sensaciones, cuya relación fue positiva- y positivas con el estilo experiencial -exceptuando la falta de perseverancia, cuya correlación fue no significativa. De esta forma, la búsqueda de sensaciones fue la única dimensión que se correlacionó positivamente con ambos estilos de pensamiento. Se indicó validez convergente al demostrar evidencia empírica sobre esta relación teorizada en estudios previos. Era esperable que el estilo de pensamiento experiencial se asociara con comportamientos impulsivos -específicamente la falta de premeditación-, al ser definido como un pensamiento automático e involucrar una reacción veloz y emocional en la resolución de problemas (Norris y Epstein, 2011). La asociación entre el factor racional y la impulsividad también ha seguido el sentido esperado al aportar consistencia empírica a la descripción de Pacini y Epstein (1999) de dicho modelo cognitivo, que caracteriza el pensamiento racional como analítico y relativamente lento y libre de afecto. Además, el procesamiento racional se indica como un factor protector ante posibles comportamientos de riesgo (Edmond y Marmurek, 2010). El resultado también presenta congruencia con un estudio previo, que asociaba mayores niveles de urgencia -ya sea positiva o negativa- a una disminución en el carácter racional de la toma de decisiones debido a un foco a corto plazo (Cyders y Smith, 2008), lo cual indicó validez de convergencia de la presente escala.

Por otra parte, el descubrimiento consistente en que la psicopatía se relaciona con la impulsividad resulta congruente con investigaciones anteriores (Crysel, Crosier y Webster, 2013; Jones y Paulhus, 2011; Malesza y Ostaszewski, 2016) que atribuyen elevados niveles de impulsividad a la psicopatía y la asocian con falta de escrupulosidad y déficits en el autocontrol (Hare, 1991).

En cuanto a las correlaciones parciales, controlando los puntajes en psicopatía, se observó que el maquiavelismo se correlacionó de forma negativa con falta de premeditación. Es importante que también se encontrara una correlación significativa y positiva con urgencia, aunque fue de valores mínimos, que no permiten concluir una asociación. De esta forma, dichos resultados son satisfactorios, al coincidir con estudios anteriores, en los que el maquiavelismo no demostró relación con la impulsividad (Jones y Paulhus (2011); Malesza y Ostaszewski, 2016). Por otro lado, difieren de los estudios de Crysel, Crosier y Webster (2013), quienes encontraron una relación entre la impulsividad y el maquiavelismo. Además, la relación negativa con falta de premeditación sigue la línea de la conceptualización de los maquiavélicos como manipuladores con autocontrol (Jones y Paulhus, 2011) que va en línea con su habilidad para realizar cálculos y desplegar estrategias a largo plazo para su propio beneficio (Vesna, Jelena y Veljko, 2019).

El narcisismo se asoció de forma positiva con la búsqueda de sensaciones y de forma negativa con la falta de perseverancia. Dichos resultados coincidieron con un estudio que concluyó que la impulsividad del narcisista se 
debe mayormente a rasgos relacionados a la extraversión, como la búsqueda de sensaciones y a la activación del comportamiento, en vez de a rasgos relacionados a la desregulación emocional o la escrupulosidad (Miller, Campbell, Young, Lakey, Reidy, Zeichner y Goodie, 2009). A su vez, los narcisistas estructuran todo su entorno social alrededor de la volatilidad con el fin de satisfacer su hedonismo y búsqueda de sensaciones (Jonason, Valentine y Harbeson, 2011). Sin embargo, aunque en el presente estudio el narcisismo se asociaría a la perseverancia, Hart, Tortoriello y Richardson (2018) resumen que los narcisistas presentan mayor resiliencia, emociones positivas y persistencia frente al fracaso solo ante la falta de formas alternativas de mantener su autoestima (Wallace, Ready y Weitenhagen, 2009).

Estos resultados indicarían que la escala de impulsividad breve mantendría adecuadas propiedades psicométricas en una muestra de adultos argentinos, pero con una estructura factorial distinta, siendo esta más similar a la versión original del instrumento. No obstante, es necesario ampliar la investigación, ya que la presente muestra fue recogida de forma intencional. Entre las limitaciones del presente estudio se encuentra la utilización de una muestra argentina, que no asegura la validez intercultural del instrumento en otros países hispanohablantes, así como el método de recolección de datos intencional y online. Además, el uso exclusivo de autoinformes implica varianza compartida debido al método de recolección de datos compartido. La utilización de un autorreporte para la evaluación de la impulsividad implica una limitación al haberse planteado que los autoinformes y las pruebas experimentales probablemente evaluarían diferentes componentes del comportamiento impulsivo (Malesza y Ostaszewski, 2016). Además, su estructura de cuatro factores deja fuera la evaluación de la urgencia positiva, cuya incorporación implicaba la diferencia de la UPPS-P con respecto al UPPS. Se debe considerar que la presente validación se ha realizado a partir de la adaptación al español en versión breve y no se han incluido la totalidad de ítems de la escala original. Por otra parte, aunque los coeficientes de consistencia interna son aceptables, implican valores menores a los de adaptaciones en otros idiomas, siendo solo similares a los de la versión española de Cándido et al. (2012). Futuras investigaciones deberían evaluar si el instrumento conserva sus propiedades psicométricas al utilizar muestras de distintas regiones de la Argentina y de otros países hispanohablantes. Sería relevante evaluar la confiabilidad test-retest, y emplear otras técnicas de recolección de datos con selección al azar, así como la utilización de otros instrumentos, además del autoinforme, como pruebas neuropsicológicas. A su vez, es recomendable examinar las propiedades de la presente adaptación en una muestra clínica, incluyéndose diversas psicopatologías relacionadas con la impulsividad, tales como trastorno de personalidad límite, bipolar, de déficit de atención con hiperactividad, trastornos alimenticios, alcoholismo, juego patológico, entre otros. Resulta relevante, a su vez, la comparación de la presente escala con otros modelos multidimensionales de impulsividad, así como con la escala con la totalidad de ítems -versión no breve- en una muestra argentina.

Bartels, D. M. (2008). Principled moral sentiment and the flexibility of moral judgment and decision making. Cognition, 108(2), 381-417. https://doi.org/10.1016/j.cognition.2008.03.001

Bentler, P. M. (1992). On the fit of models to covariances and methodology to the Bulletin. Psychological Bulletin, 112(3), 400. https://doi.org/10.1037/0033-2909.112.3.400

Billieux, J., Rochat, L., Ceschi, G., Carré, A., Offerlin-Meyer, I., Defeldre, A. C. y Van der Linden, M. (2012). Validation of a short French version of the UPPS-P Impulsive Behavior Scale. Comprehensive Psychi- 
atry, 53(5), 609-615. https://doi.org/10.1016/j. comppsych.2011.09.001

Boomsma, A. y Hoogland, J. J. (2001). The robustness of LISREL modeling revisited. En R. Cudeck, S. du Toit y D. Sörbom (Eds.), Structural equation models: present and future. A festschrift in honor of Karl Jöreskog (pp. 139-168). Lincolnwood, IL: Scientific Software International.

Buss, A. H. y Plomin, R. (1975). A temperament theory of personality development. New York, NY: Wiley.

Byrne, B. M. (1989). A primer of LISREL: Basic Applications and programming for confirmatory factor analytic models. New York, NY: Springer-Verlag

Byrne, B. (2010). Structural equation modeling with AMOS: Basic concepts, applications, and programming. Nueva York, NY: Routledge. https://doi.org/10.4324/9780203805534

Cándido, A., Orduña, E., Perales, J. C., Verdejo-García, A. y Billieux, J. (2012). Validation of a short Spanish version of the UPPS-P impulsive behavior scale. Trastornos Adictivos, 14(3), 73-78. https://doi.org/10.1016/ S1575-0973(12)70048-X

Claes, L., Vandereycken, W. y Vertommen, H. (2005). Impulsivity-related traits in eating disorder patients. Personality and Individual Differences, 39(4), 739-749. https://doi. org/10.1016/j.paid.2005.02.022

Costa, P. T. y McCrae, R. R. (1992). Revised NEO Personality Inventory (NEO-PI-R) and NEO Five-Factor Inventory professional manual. Odessa, FL: Psychological Assessment Resources.

Crysel, L. C., Crosier, B. S. y Webster, G. D. (2013). The Dark Triad and risk behavior. Personality and Individual Differences, 54(1), 35-40. https://doi.org/10.1016/j. paid.2012.07.029

Cupani, M. (2012). Análisis de Ecuaciones Estructurales: conceptos, etapas de desarrollo y un ejemplo de aplicación. Tesis, 1, 186-199

Curry, I., Luk, J. W., Trim, R. S., Hopfer, C. J., Hewitt, J. K., Stallings, M. C., ... y Wall, T. L. (2018). Impulsivity dimensions and risky sex behaviors in an at-risk young adult sample. Archives of Sexual Behavior, 47(2), 529-536. https://doi.org/10.1007/s10508-0171054-x

Cyders, M. A., Littlefield, A. K., Coffey, S. y Karyadi, K. A. (2014). Examination of a short English version of the UPPS-P Impulsive Behavior Scale. Addictive Behaviors, 39(9), 1372-1376. https://doi.org/10.1016/j. addbeh.2014.02.013

Cyders, M. A. y Smith, G. T. (2008). Emotionbased dispositions to rash action: positive and negative urgency. Psychological Bulletin, 134(6), 807. https://doi.org/10.1037/ a0013341

Cyders, M.A., Smith, G. T., Spillane, N. S., Fischer, S., Annus, A. M. y Peterson, C. (2007). Integration of impulsivity and positive mood to predict risky behavior: Development and validation of a measure of positive urgency. Psychological Assessment, 19(1), 107-118. https://doi. org/10.1037/1040-3590.19.1.107

Czibor, A., Vincze, O. y Bereczkei, T. (2014). Feelings and motives underlying Machiavellian behavioural strategies; narrative reports in a social dilemma situation. International Journal of Psychology, 49(6), 519-524. https:// doi.org/10.1002/ijop.12077

Dickman, S. J. (1990). Functional and dysfunctional impulsivity: Personality and cognitive correlates. Journal of Personality and Social Psychology, 58(1), 95-102. https://doi. org/10.1037/0022-3514.58.1.95

Djeriouat, H. y Trémolière, B. (2014). The Dark Triad of personality and utilitarian moral judgment: The mediating role of Honesty/Humility and Harm/Care. Personality and Individual Differences, 67, 11-16. https://doi.or/10.1016/j. paid.2013.12.026

D’Orta, I., Burnay, J., Aiello, D., Niolu, C., Siracusano, A., Timpanaro, L., ...y Billieux, J. (2015). Development and validation of a short Italian UPPS-P Impulsive Behavior Scale. Addictive Behaviors Reports, 2, 19-22. https://doi. org/10.1016/j.abrep.2015.04.003

Edmond, M. S. y Marmurek, H. H. C. (2010). Gambling related cognitions mediate the asso- 
ciation between thinking style and problem gambling severity. Journal of Gambling Studies, 26, 257-267. https://doi.org/10.1007/ s10899-009-9164-6

Fehm, L. y Hoyer, J. (2004). Measuring thought control strategies: The thought control questionnaire and a look beyond. Cognitive Therapy and Research, 28(1), 105-117. https://org. doi/10.1023/B:COTR.0000016933.41653.dc

Furnham, A., Richards, S. C. y Paulhus, D. L. (2013). The Dark Triad of Personality: A 10 Year Review. Social and Personality Psychology Compass, 7(3), 199-216. https:// doi.org/10.1111/spc3.12018

Hahn, A. M., Simons, R. M. y Hahn, C. K. (2016). Five factors of impulsivity: Unique pathways to borderline and antisocial personality features and subsequent alcohol problems. Personality and Individual Differences, 99, 313-319. https://doi.org/10.1016/j.paid.2016.05.035

Hare, R. D. (1991). The Psychopathy Checklist-Revised (PCL-R). Toronto, ON: Multi Health Systems.

Hart, W., Richardson, K. y Tortoriello, G. K. (2018). Narcissists stand united: Grandiose and vulnerable narcissists agree that others' narcissism is less repulsive. Journal of Individual Differences, 39(4), 204-211. https://doi. org/10.1027/1614-0001/a000265

Horn, J. L. (1965). A rationale and test for the number of factors in factor analysis. Psychometrika, 30(2), 179-185.

Hu, L. T. y Bentler, P. M. (1999). Cutoff criteria for fit indexes in covariance structure analysis: Conventional criteria versus new alternatives. Structural Equation Modeling: a Multidisciplinary Journal, 6(1), 1-55. https://doi. org/10.1080/10705519909540118

Jonason, P. K. y Tost, J. (2010). I just cannot control myself: The Dark Triad and self-control. Personality and Individual Differences, 49(6), 611-615. https://doi. org/10.1016/j.paid.2010.05.031

Jonason, P. K., Valentine, K. A., Li, N. P. y Harbeson, C. L. (2011). Mate-selection and the Dark Triad: Facilitating a short-term mating strategy and creating a volatile envi- ronment. Personality and Individual Differences, 51, 759-763. https://doi.org/10.1016/j. paid.2011.06.025

Jones, D. N. y Paulhus, D. L. (2011). The role of impulsivity in the Dark Triad of personality. Personality and Individual Differences, 51(5), 679-682. https://doi. org/10.1016/j.paid.2011.04.011

Jones, D. N. y Paulhus, D. L. (2014). Introducing the short dark triad (SD3) a brief measure of dark personality traits. Assessment, 21(1), 28-41. https://doi.org/10.1177/1073191113514105

Lorenzo-Seva, U. (2013). Why rotate my data using Promin? Technical Report. Department of Psychology, Universitat Rovira i Virgili, Tarragona, España.

Lorenzo-Seva, U. y Ferrando, P. J. (2013). FACTOR 9.2: A Comprehensive Program for Fitting Exploratory and Semiconfirmatory Factor Analysis and IRT Models. Applied Psychological Measurement, 37(6), 497-498. https://doi.org/10.1177/0146621613487794

Lynam, D. R., Smith, G. T., Whiteside, S. P. y Cyders, M. A. (2006). The UPPS-P: Assessing five personality pathways to impulsive behavior. West Lafayette, Indiana: Purdue University.

Magid, V., MacLean, M. G. y Colder, C. R. (2007). Differentiating between sensation seeking and impulsivity through their mediated relations with alcohol use and problems. Addictive Behaviors, 32(10), 2046-2061. https://doi. org/10.1016/j.addbeh.2007.01.015

Malesza, M. y Ostaszewski, P. (2016). Dark side of impulsivity - Associations between the Dark Triad, self-report and behavioral measures of impulsivity. Personality and Individual Differences, 88, 197-201. https://doi.org/10.1016/j. paid.2015.09.016

Miller, J. D., Campbell, W. K., Young, D. L., Lakey, C. E., Reidy, D. E., Zeichner, A. y Goodie, A. S. (2009). Examining the Relations Among Narcissism, Impulsivity, and Self-Defeating Behaviors. Journal of Personality, 77(3), 761-794. https://doi.org/10.1111/ j.1467-6494.2009.00564.X

Nogueira Sediyama, C. Y. (2014). Investigação 
das características psicométricas da UPPS Escala de Comportamento Impulsivo para uma população brasileira (Tesis de Maestria). Universidade Federal de Minas Gerais Universidade Federal de Minas Gerais, Brasil.

Nohales Nieto, B. (2015). La tríada oscura de la personalidad: adaptación al español de los cuestionarios Dirty dozen y Short dark triad (Tesis de Grado). Universitat Jaume I, España. https://doi.org/10.6035/AgoraSalut.2017.4.28

Norris, P. y Epstein, S. (2011). An experiential thinking style: Its facets and relations with objective and subjective criterion measures. Journal of Personality, 79(5), 1043-1080. https://doi. org/10.1111/j.1467-6494.2011.00718.x

O'Connor, B. P. (2000). SPSS and SAS programs for determining the number of components using parallel analysis and Velicer's MAP test. Behavior Research Methods, Instruments, \& Computers, 32(3), 396-402. https://doi. org/10.3758/bf03200807

Pacini, R. y Epstein, S. (1999). The relation of rational and experiential information processing styles to personality, basic beliefs, and the ratio-bias phenomenon. Journal of Personality and Social Psychology, 76(6), 972. https://doi.org/10.1037/0022-3514.76.6.972

Paulhus, D. L., y Williams, K. M. (2002). The dark triad of personality: Narcissism, Machiavellianism, and psychopathy. Journal of research in personality, 36(6), 556-563. https:// doi.org/10.1016/S0092-6566(02)00505-6

Patton, J. H., Stanford, M. S. y Barratt, E. S. (1995). Factor structure of the Barratt impulsiveness scale. Journal of Clinical Psychology, 51(6), 768-774. $\quad$ https://doi.org/10.1002/10974679(199511)51:6<768::AIDJCLP2270510607>3.0.CO;2-1

Pérez, E. J. P. (2009). Evaluación de la impulsividad funcional y disfuncional en adictos a sustancias mediante el Inventario de Dickman. Psicothema, 21(4), 585-591.

Reyna, C.y Ortiz, M. V.(2016). Psychometric study of the Rational Experiential Inventory among undergraduate Argentinean students. Revista de Psicología, 34(2), 337-355. https://doi. org/10.18800/psico.201602.005
Riding, R. J. y Rayner S. (2000). International Perspectives on Individual Differences, vol. 1: Cognitive Styles. New York, NY: Ablex

Savvidou, L. G., Fagundo, A. B., Fernández-Aranda, F., Granero, R., Claes, L., Mallorquí-Baqué, N., ...y del Pino-Gutiérrez, A. (2017). Is gambling disorder associated with impulsivity traits measured by the UPPS-P and is this association moderated by sex and age? Comprehensive Psychiatry, 72, 106-113. https://doi.org/10.1016/j. comppsych.2016.10.005

Schmidt, R. E., Gay, P., d'Acremont, M. y Van der Linden, M. (2008). A German adaptation of the UPPS Impulsive Behavior Scale: Psychometric properties and factor structure. Swiss Journal of Psychology / Schweizerische Zeitschriftü̈r Psychologiel Revue Suisse de sychologie, 67(2), 107. https://doi. org/10.1024/1421-0185.67.2.107

Stanford, M. S., Mathias, C. W., Dougherty, D. M., Lake, S. L., Anderson, N. E. y Patton, J. H. (2009). Fifty years of the Barratt Impulsiveness Scale: An update and review. Personality and Individual Differences, 47(5), 385-395. https://doi.org/10.1016/j.paid.2009.04.008

Steinberg, L., Albert, D., Cauffman, E., Banich, M., Graham, S. y Woolard, J. (2008). Age differences in sensation seeking and impulsivity as indexed by behavior and self-report: Evidence for a dual systems model. Developmental Psychology, 44(6), 1764-1778. https:// doi.org/10.1037/a0012955

Wells, A. y Davies, M. I. (1994). The Thought Control Questionnaire: A measure of individual differences in the control of unwanted thoughts. Behavior Research and Therapy, 32(8), 871-878. https://doi. org/10.1016/0005-7967(94)90168-6

Weston, R. y Gore Jr, P. A. (2006). A brief guide to structural equation modeling. The Counseling Psychologist, 34(5), 719-751. https:// doi.org/10.1177/0011000006286345

Whiteside, S. P. y Lynam, D. R. (2001). The five factor model and impulsivity: Using a structural model of personality to understand impulsivity. Personality and Individual Differ- 
ences, 30(4), 669-689. https://doi.org/10.1016/ S0191-8869(00)00064-7

Whiteside, S. P. y Lynam, D. R. (2003). Understanding the role of impulsivity and externalizing psychopathology in alcohol abuse: application of the UPPS impulsive behavior scale. Experimental and Clinical Psychopharmacology, 11(3), 210. https://doi. org/10.1037/1064-1297.11.3.210

Whiteside, S. P., Lynam, D. R., Miller, J. D. y Reynolds, S. K. (2005). Validation of the UPPS impulsive behaviour scale: a four-factor model of impulsivity. European Journal of Personality: Published for the European Association of Personality Psychology, 19(7), 559-574. https://doi.org/10.1002/per.556

Vazire, S. y Funder, D. C. (2006). Impulsivity and the self-defeating behavior of narcissists. Personality and Social Psychology Review, 10(2), 154-165. https://doi. org/10.1207/s15327957pspr1002_4

Verdejo-García, A., Bechara, A., Recknor, E. C. y Pérez-García, M. (2007).Negative emotion-driven impulsivity predicts substance dependence problems. Drug and Alcohol Dependence, 91(2-3), 213-219. https://doi. org/10.1016/j.drugalcdep.2007.05.025

Verdejo-García, A., Lozano, Ó., Moya, M., Alcázar, M. A. y Pérez-García, M. (2010). Psychometric properties of a spanish version of the UPPS-P impulsive behavior scale: reliability, validity and association with trait and cognitive impulsivity. Journal of Personality Assessment, 92(1), 70-77. https://doi. org/10.1080/00223890903382369

Vesna, G., Jelena, D. y Veljko, Đ. (2019). The Dark Triad, Amorality, and Impul- sivity. Psihologija, 52(1).

Wallace, H. M., Ready, C. B. y Weitenhagen, E. (2009). Narcissism and Task Persistence. Self and Identity, 8(1), 78-93. https://doi. org/10.1080/15298860802194346

Zuckerman, M. (1979). Beyond the optimal level of arousal. Hillsdale, NJ: Lawrence Erlbraum Associates.

Zuckerman, M. (2002). Zuckerman-Kuhlman personality questionnaire (ZKPQ): An alternative five-factorial model. Big Five Assessment, 377-396.

Zuckerman, M. (2008). Zuckerman-Kuhlman Personality Questionnaire: An operational definition of the alternative five factorial model of personality. En G.J. Boyle, G. Matthews, y D.H. Saklofske (Eds.), The SAGE handbook of personality theory and assessment, Vol. 2: Personality measurement and testing (pp. 219-238). Los Angeles, CA: Sage.

Zuckerman, M. (2010). Sensation Seeking. En I. B Weiner y W.E Craighead (Eds), The Corsini Encyclopedia of Psychology. Hoboken, NJ: John Wiley \& Sons, Inc, 1-4. https://doi.org/ 10.1002/9780470479216.corpsy0843

Zuckerman, M., Eysenck, S. B. y Eysenck, H. J. (1978). Sensation seeking in England and America: Cross-cultural, age, and sex comparisons. Journal of Consulting and Clinical Psychology, 46(1), 139-149. https://doi. org/10.1037/0022-006X.46.1.139

Zuckerman, M., Kuhlman, D. M., y Camac, C. (1988). What lies beyond E and N? Factor analyses of scales believed to measure basic dimensions of personality. Journal of Personality and Social Psychology, 54(1), 96. https:// doi.org/10.1037/0022-3514.54.1.96

Recibido: 11 de mayo de 2020 Aceptado: 27 de agosto de 2021 